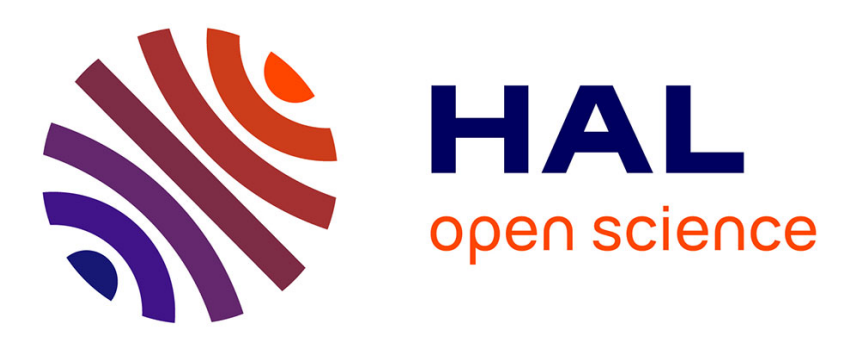

\title{
Management stratégique et dynamiques d'appropriation des outils de gestion: proposition d'une grille de lecture
} Gaelle Dechamp, Hervé Goy, Amaury Grimand, François-Xavier de Vaujany

\section{To cite this version:}

Gaelle Dechamp, Hervé Goy, Amaury Grimand, François-Xavier de Vaujany. Management stratégique et dynamiques d'appropriation des outils de gestion: proposition d'une grille de lecture. Revue management \& avenir, 2006, 9, pp.181-200. halshs-00675773

\section{HAL Id: halshs-00675773 \\ https://shs.hal.science/halshs-00675773}

Submitted on 11 Apr 2012

HAL is a multi-disciplinary open access archive for the deposit and dissemination of scientific research documents, whether they are published or not. The documents may come from teaching and research institutions in France or abroad, or from public or private research centers.
L'archive ouverte pluridisciplinaire HAL, est destinée au dépôt et à la diffusion de documents scientifiques de niveau recherche, publiés ou non, émanant des établissements d'enseignement et de recherche français ou étrangers, des laboratoires publics ou privés. 


\title{
Management stratégique et dynamiques d'appropriation des outils de gestion : proposition d'une grille de lecture.
}

\author{
par Gaëlle Dechamp, \\ Hervé Goy, \\ Amaury Grimand \\ et François-Xavier de Vaujany
}

Résumé De tous les champs disciplinaires de la gestion, celui abordant le point de vue du stratège est potentiellement l'un des plus délicats à aborder s'agissant de l'étude de l'appropriation des dispositifs et autres outils de conception et de pilotage stratégiques. Mais passée cette première réflexion qui tient à la nature même du management stratégique (nécessairement transversale et englobante), rien ne permet de présager de l'homogénéité des dispositifs stratégiques et de leur appropriation par les acteurs de l'organisation. Comme premier élément d'analyse, nous proposons de distinguer un management stratégique de nature endogène d'un management d'une nature plus exogène. Envisagé sous l'angle d'un nouveau couple ago-antagoniste, nous défendons l'idée selon laquelle cette dichotomie permet d'établir un cadre d'analyse pertinent pour l'étude des dynamiques appropriatives des dispositifs de gestion stratégique.

Abstract Among all the disciplinary fields of management, strategy is potentially one of the most delicate to approach concerning the study of appropriation of management tools. Beyond this first reflexion holding with the nature of strategic management (necessarily transverse and including), predicting homogeneity of strategic devices and their appropriation by the members of an organization remains complicated. We thus propose to distinguish an endogenous strategic management of an exogenous one. As a new ago-antagonist couple, we believe that this dichotomy makes possible the establishment of a relevant framework for the study of appropriative dynamics of strategic management tools. 
L'étude de l'appropriation des outils de gestion n'a de sens pour nous que si les processus d'appropriation potentiellement observables sont envisagés comme intégrés à une démarche plus globale de conception de l'action collective. C'est en ce sens que de Vaujany (2005) définit trois regards sur la question de l'appropriation :

\begin{tabular}{|c|c|c|c|}
\hline & $\begin{array}{l}\text { Nature des objets } \\
\text { et outils de gestion }\end{array}$ & $\begin{array}{l}\text { Nature du processus } \\
\text { d'appropriation }\end{array}$ & $\begin{array}{l}\text { Fondements } \\
\text { théoriques }\end{array}$ \\
\hline $\begin{array}{l}\text { Perspective } \\
\text { rationnelle }\end{array}$ & $\begin{array}{l}\text { Un vecteur de rationalisation, } \\
\text { un outil de travail }\end{array}$ & $\begin{array}{l}\text { Un processus normalisé, } \\
\text { l'appropriation } \\
\text { est "instantanée" }\end{array}$ & $\begin{array}{l}\text { Théorie micro-économique } \\
\text { classique } \\
\text { Théories fayoliennes } \\
\text { et tayloriennes }\end{array}$ \\
\hline $\begin{array}{l}\text { Perspective } \\
\text { socio-politique }\end{array}$ & $\begin{array}{l}\text { Un outil de valorisation une } \\
\text { "médaille"), de rhétorique } \\
\text { un "argument") ou } \\
\text { d'influence un "atout") }\end{array}$ & $\begin{array}{l}\text { Un acte social, l'appropriation } \\
\text { est un processus collectif qui } \\
\text { s'inscrit dans la durée }\end{array}$ & $\begin{array}{l}\text { Sociologie des organisations } \\
\text { Crozier et Friedberg, } 1977 \text {; } \\
\text { Sainsaulieu, 1998) } \\
\text { Sociologie générale } \\
\text { Bourdieu, 2000 ; Giddens, } \\
1984 \text {; Archer, 1995) } \\
\end{array}$ \\
\hline $\begin{array}{l}\text { Perspective } \\
\text { psycho- } \\
\text { cognitive }\end{array}$ & $\begin{array}{l}\text { Un support d'apprentissage, } \\
\text { un objet affectif ou un objet } \\
\text { de traitement de l'information }\end{array}$ & $\begin{array}{l}\text { Un processus psycho-cognitif, } \\
\text { l'appropriation est un processus } \\
\text { individuel voire collectif) qui } \\
\text { s'inscrit dans la durée }\end{array}$ & $\begin{array}{l}\text { Théorie de la rationalité } \\
\text { limitée Simon, 1965) } \\
\text { Psychologie cognitive } \\
\text { Piaget, 1967, 1975) } \\
\text { Perspective psychanalytique } \\
\text { Pagès et al, 1992) }\end{array}$ \\
\hline
\end{tabular}

Tableau 1 : Trois regards sur l'appropriation

Cette perspective appropriative a une résonance toute particulière dans le champ de la stratégie, dans la mesure où elle permet d'envisager une approche à la fois transversale et englobante des dispositifs stratégiques et de leur appropriation par les différents acteurs de l'entreprise. On peut en effet s'interroger sur les points suivants : n'y a-t-il pas des éléments de cette théorie de l'appropriation spécifiques à certaines logiques et certains contextes du management stratégique ? En outre, les membres du noyau stratégique ${ }^{120}$ n'actionnent-ils pas des objets de nature différente selon qu'ils sont en phase de création, de crise ou de "normalité" stratégique, selon que le projet stratégique est en devenir, en question ou constitue un statu quo pour la majorité des acteurs de l'organisation ? Comme premier élément de réponse à ces interrogations, nous proposons de distinguer un management stratégique de nature endogène d'un management d'une nature plus exogène.

Un des points de départ de cette dichotomie provient de l'opposition "eventbased" - "time-based" suggérée par Gersick (1994). Au terme d'une monographie sur une jeune société de capital-risque, l'auteur a proposé deux modalités de gestion stratégique permettant de traiter "la tension entre la persévérance et la flexibilité". Dans le cas d'une gestion stratégique basée sur le temps, ce sont les

${ }^{120}$ Selon Martinet (1984), "nous appellerons noyau stratégique le groupe (composé de 1 à n individus) qui a, de part la propriété et/ou le mandat, le pouvoir d'émettre des stratégies favorables aux buts de l'institution [...]. Il peut s'enrichir en s'agrégeant des individus puisés dans l'organisation (cadres...) ou à l'extérieur (experts, conseils, " alliés »)". 


\section{Management stratégique et dynamiques d'appropriation des outils de gestion : proposition d'une grille de lecture.}

échéances qui guident l'action managériale. L'auteur remarque également une tendance naturelle à la réorientation à mi-parcours des projets, passée une première phase d'enracinement stratégique ${ }^{121}$. La dynamique même du pilotage est donc ici plutôt endogène. Dans le cas d'une gestion stratégique basée sur les événements, les réorientations sont davantage amenées et légitimées par les diverses situations qui ponctuent la vie de l'entreprise et le déploiement de ses projets. Le pilotage, son orientation, son rythme, sont ici davantage marqués par des mécanismes exogènes, en particulier le dépassement de seuils financiers conventionnels ou encore des évolutions de l'environnement commercial et technologique.

En croisant le travail de Gersick (1994) avec la perspective appropriative présentée ci-dessus, nous suggérons un continuum stratégique plus large basé sur l'opposition management stratégique endogène et management stratégique exogène, différenciables par les éléments qui les induisent, le statut du noyau stratégique ainsi que le statut des objets de gestion manipulés. Cette approche nous conduit par conséquent à envisager managements stratégiques exogène et endogène comme deux idéaux-types qui constituent les extrêmes d'un continuum. On pourrait résumer notre propos au moyen du tableau suivant :

\begin{tabular}{|l|l|l|}
\hline & Management stratégique exogène & Management stratégique endogène \\
\hline $\begin{array}{l}\text { Inducteurs } \\
\text { et supports } \\
\text { du mode } \\
\text { de gestion }\end{array}$ & $\begin{array}{l}\text { Moment "chauds" qui rendent inévitable la } \\
\text { réflexion stratégique : crises ou phase de création ; } \\
\text { La logique stratégique est "interpellée" et } \\
\text { structurée par l'environnement institutionnel : } \\
\text { les tribunaux decommerce, les organismes de } \\
\text { formation à la gestion,les banques, les fournisseurs } \\
\text { et créanciers, les syndicats de l'entreprise... } \\
\text { Les événements plus ou moins mis en scène } \\
\text { par le noyau stratégique) sont au cœur du } \\
\text { mode de gestion. }\end{array}$ & $\begin{array}{l}\text { lénau stratégique visent à adapter } \\
\text { construire le potentiel stratégique ; } \\
\text { Le temps est au cœur du mode de gestion. }\end{array}$ \\
\hline $\begin{array}{l}\text { Statut du } \\
\text { noyau } \\
\text { stratégique }\end{array}$ & $\begin{array}{l}\text { Qu'il s'agisse du pré-noyau stratégique de } \\
\text { l'entreprise en création ou bien du noyau } \\
\text { stratégique de l'entreprise en crise, dans les deux } \\
\text { cas les acteurscorrespondent à une régulation } \\
\text { autonome. Ils doivent se conformer, contourner } \\
\text { ou transformer les règles promues par des } \\
\text { régulations de contrôle institutionnelles. }\end{array}$ & $\begin{array}{l}\text { Les acteurs du noyau stratégique correspondent } \\
\text { ici davantage à une régulation de contrôle. } \\
\text { Ils mettent en avant des règles de gestion que } \\
\text { les acteurs autonomes de l'organisation } \\
\text { pourront ensuite suivre, transformer ou éviter. }\end{array}$ \\
\hline $\begin{array}{l}\text { Statut des objets } \\
\text { de gestion manipulés }\end{array}$ & \begin{tabular}{l} 
Plutôt des objets institutionnels et sociétaux \\
\hline
\end{tabular} & Plutôt des objets organisationnels \\
\hline
\end{tabular}

Tableau 2 : Comparaison entre management stratégique endogène et exogène

${ }^{121}$ Cela prend la forme à l'arrivée de ce qui est considéré par les membres d'un groupe comme la mi-parcours. Gersick (1994, p.12) explique ainsi que "Sometimes consciously, sometimes not, group select the midpoint (or occasionally another time) as a heuristic milestone and use it like an alarm clock, to help ensure they will move fast enough to finish their deadlines. In effect, groups allow themselves to persist with opening work patterns until the midpoint. The event of reaching the midpoint - not the completion of a given amount of work-stimulates feelings of urgency and an awareness of the deadline and breaks groups' momentum. Groups feel it is time to move ahead". 
L'étude des mécanismes d'appropriation des objets de gestion liés à chacune de ces perspectives ouvre sans nul doute des problématiques de recherche fécondes. Nous défendons en ce sens l'idée selon laquelle la distinction entre management stratégique endogène et exogène constitue une grille de lecture pertinente pour l'étude de l'appropriation des dispositifs stratégiques de gestion des organisations. Sans ambitionner pour autant de traiter chacun de ces deux volets dans le cadre de la présente contribution, nous centrerons notre propos sur les dynamiques d'appropriation liées à la perspective endogène de la stratégie ${ }^{122}$. C'est pourquoi, nous développerons dans une première partie les trois trajectoires que nous proposons d'emprunter dans l'analyse des dynamiques d'appropriation des outils de gestion en stratégie. Ce premier parcours engagera dans un second temps une réflexion sur les moteurs endogènes du management stratégique. En particulier, pour le cas du noyau stratégique qui manipule les objets de gestion sociétaux ou organisationnels, nous nous demanderons s'il n'existe pas des processus d'appropriation spécifiques. Nous verrons également si, notamment en terme de légitimité, pour les acteurs périphériques au noyau stratégique, il n'existe pas des mécanismes d'appropriation propres à chaque type d'objet manipulé par le noyau stratégique.

\section{Trois regards sur l'appropriation des dispositifs stratégiques}

Cette première partie ambitionne d'engager une réflexion sur les éléments individuels (ex : biais cognitifs, cartes cognitives, effet d'enracinement et "effet middle point", groupaux ex : cartes cognitives) ou organisationnels (ex : paradigme stratégique et culture d'entreprise) qui donnent une certaine "persévérance" à l'action stratégique, et génèrent parfois des mécanismes endogènes de changement stratégique. Trois "angles de vue" nous semblent ainsi possibles.

La perspective psycho-cognitive s’intéresse à la façon dont les acteurs construisent une intelligence des situations stratégiques. L'appropriation est ici entendue comme le processus d'acquisition de nouvelles connaissances par des acteurs, capables et désireux de les exploiter pour améliorer les processus de décision, ou influencer d'autres acteurs organisationnels (Miller, 1996). Règles, dispositifs, outils et objets de gestion sont dans cette perspective envisagés comme autant de supports potentiels d'apprentissage. L'appropriation emprunte également un registre symbolique et mobilise des objets de gestion permettant un investissement affectif. Ainsi, les grandes institutions ont-elles besoin de mythes fondateurs ${ }^{123}$ qui lui

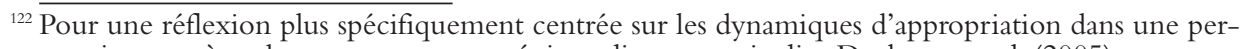
spective exogène du management stratégique, lire en particulier Dechamp et al. (2005).

${ }^{123}$ Ainsi, l'une des premières décisions de Carly Fiorina, lorsqu'elle prit la tête de Hewlett-Packard en 1999 fut de racheter le célèbre "garage" où les deux fondateurs, Bill Hewlett et Dave Packard, inventèrent un oscillateur audio qui servira par la suite à sonoriser les premiers films Disney.(...)
} 


\section{Management stratégique et dynamiques d'appropriation des outils de gestion : proposition d'une grille de lecture.}

donnent sens et la légitiment. Ce système managinaire (Aubert et De Gaulejac, 1991) est producteur de névroses et de pathologies, et conditionne la trajectoire "appropriative" des acteurs. Ces derniers vont ainsi être enclins à user des règles, dispositifs, outils et objets de gestion comme autant de mécanismes collectifs de défense contre l'anxiété.

Une perspective socio-politique amène à envisager l'appropriation comme résultant du jeu des acteurs, de leur capacité à saisir des marges d'autonomie, à créer des coalitions. A ce titre, l'appropriation parait particulièrement adaptée au champ de la stratégie, l'environnement stratégique d'une entreprise étant aussi un environnement d'acteurs et de parties prenantes de toutes sortes : fournisseurs, concurrents, clients, actionnaires, syndicats, groupes de lobbying... Les objets de gestion, dans cette perspective, sont appropriés ou plus exactement réappropriés par les acteurs, à des fins de valorisation personnelle, comme argument rhétorique permettant de légitimer leurs décisions et actions, individuelles ou collectives, ou comme support d'influence.

Dans une approche rationnelle, l'appropriation s'inscrit davantage dans une vision mécaniste de l'organisation (Morgan, 1997), totalement centrée sur la régulation de contrôle. Par conséquent, la régulation n'a pas ou plutôt s'imagine ne pas avoir de limites cognitives ou politiques à son action. On se positionne dans un cadre théorique où les problèmes de légitimité, de biais cognitifs, d'affects... sont absents des processus d'appropriation des objets liés à la stratégie, soit parce que l'organisation se limite finalement à l'entrepreneur rationnel qui la porte, soit parce qu'elle est un "corps" qui suit de façon automatique les instructions de la "tête".

Les trois perspectives "appropriatives" précédentes, et les courants théoriques afférents, sont résumés dans le tableau ci-dessous, en distinguant les acteurs impliqués dans le processus (noyau stratégique, périphérie ou interaction noyau/périphérie). Relativement à l'acception du concept de noyau stratégique tel que Martinet (1984) l'a formulée, et que nous avons rappelée en introduction, la périphérie peut s'envisager comme l'ensemble des acteurs extérieurs au noyau, et susceptibles de s'approprier les stratégies proposées par le noyau stratégique, ou d'influencer la dynamique d'appropriation des stratégies des autres acteurs.

Une fois spécifiées, en quoi ces trois perspectives engagent-elles des dynamiques appropriatives spécifiques ou plus simplement différentes) des outils et dispositifs de gestion propres à la stratégie d'entreprise ? Qu'apporte la caractérisation endogène du management stratégique à la compréhension des mécanismes d'appropriation ? La seconde partie de cet article vise à soumettre quelques éléments de réponse à ces questionnements centraux.

(...) Toile de fonds de sa campagne de publicité mondiale, le "mythe du garage" devait ainsi lui permettre de réaffirmer le nécessaire retour à une logique d'innovation. 


\begin{tabular}{|l|l|l|l|}
\hline & Perspective psycho-cognitive & Perspective socio-politique & Perspective rationnelle \\
\hline $\begin{array}{l}\text { Noyau } \\
\text { Stratégique }\end{array}$ & $\begin{array}{l}\text { Courant de l'apprentissage organisationnel : } \\
\text { routines défensives des managers (Argyris, } \\
\text { 1995), cartographie cognitive (Cosette, } \\
\text { 1994; Huff, 1990), approche } \\
\text { psychanalytique des organisations, leadership } \\
\text { et névroses organisationnelles (Kets de Vries } \\
\text { et Miller, 1985; Enriquez, 1992) }\end{array}$ & $\begin{array}{l}\text { Gouvernement d'entreprise } \\
\text { (Charreaux, 1997) }\end{array}$ & $\begin{array}{l}\text { Management scientifique } \\
\text { (Taylor, 1913), fonction } \\
\text { administrative (Fayol, 1916), } \\
\text { théorie des coûts de } \\
\text { transaction (Coase, 1937; } \\
\text { Williamson, 1975) }\end{array}$ \\
\hline Périphérie & $\begin{array}{l}\text { Théorie des communautés de pratique } \\
\text { (Wenger et Snyder, 2000; Brown et } \\
\text { Duguid, 1991), représentations sociales } \\
\text { de la stratégie (Mounoud, 2001; } \\
\text { Lauriol, 1995) }\end{array}$ & $\begin{array}{l}\text { Analyse stratégique (Crozier et } \\
\text { Friedberg, 1977), sociologie de la } \\
\text { contrainte (Courpasson, 2000), } \\
\text { dialectique innovateurs-légalistes } \\
\text { (Alter, 1990), approche par les } \\
\text { conventions ou les “mondes sociaux" } \\
\text { de l'entreprise (Sainsaulieu et al., 1998) }\end{array}$ & $\begin{array}{l}\text { Courants de la planification } \\
\text { stratégique (Martinet, 2001), } \\
\text { recherche opérationnelle } \\
\text { (Roy, 1997) }\end{array}$ \\
\hline $\begin{array}{l}\text { Noyau } \\
\text { Stratégique } \\
+ \\
\text { Périphérie }\end{array}$ & $\begin{array}{l}\text { Courant narratif de la stratégie (Giroux et } \\
\text { Demers, 1998) : conversations stratégiques, } \\
\text { grammaire stratégique (Chanal et al., 2001), }\end{array}$ & $\begin{array}{l}\text { Régulation par la culture (Johnson } \\
\text { et Scholes, 2002 ; Schein, 1985) }\end{array}$ & $\begin{array}{l}\text { Concept de tension } \\
\text { créatrice (Senge, 1990) }\end{array}$ \\
$\begin{array}{l}\text { Simons, 1994), Routines organisationnelles } \\
\text { (Nelson et Winter, 1982) }\end{array}$ & & \\
\hline
\end{tabular}

Tableau 3 : les dynamiques appropriatives endogènes du management stratégique

\section{Quelques éléments de réflexion sur les moteurs endogènes du management stratégique}

Les trois perspectives présentées en première partie engagent une réflexion sur trois dynamiques d'appropriation des dispositifs stratégiques, que nous proposons de développer ci-après ${ }^{124}$.

\subsection{L'appropriation comme processus psycho-cognitif individuel ou collectif) qui s'inscrit dans la durée}

S'agissant du noyau stratégique, et à l'exception des théoriciens du choix stratégique (Ansoff, 1979 ; Child, 1997 et Porter, 1985) mettant en exergue une perspective volontariste et une latitude des décideurs dans leurs choix stratégiques ${ }^{125}$,

${ }^{124}$ Des propositions sur les modes de formation et d'appropriation des stratégies endogènes ont été formulées par les auteurs dans un chapitre d'ouvrage qui est la version développée de cet article (Cf. Dechamp et al., 2005). Pour des raisons formelles, ces propositions ne peuvent être reprises ici.

${ }^{125}$ L'appropriation de la stratégie engage ici un processus d'apprentissage intentionnel et analytique. Il s'appuie sur des dispositifs permanents de collecte d'informations à l'intérieur comme à l'extérieur de l'entreprise. Cette analyse proactive de l'environnement, qui mobilise largement une logique déductive, voire des techniques d'optimisation, permet de faire émerger les problématiques et opportunités stratégiques majeures et d'arbitrer au sein d'une large variété de scénarios stratégiques. Cette démarche trouve à s'incarner dans des plans stratégiques et plans opérationnels mais semble de fait exclure les autres acteurs organisationnels des processus de formation de la stratégie. 


\section{Management stratégique et dynamiques d'appropriation des outils de gestion : proposition d'une grille de lecture.}

le processus d'appropriation doit être envisagé ici comme un processus d'apprentissage, selon la dialogique assimilation/accommodation mise en évidence par Piaget $(1967,1975)$ :

- assimilation, c'est-à-dire la façon dont l'individu s'approprie en les transformant règles, dispositifs, outils et objets de gestion, de façon à les rendre compatibles avec ses propres schèmes cognitifs ;

- accommodation, c'est-à-dire la modification des schèmes cognitifs de l'individu afin de permettre l'incorporation de règles, dispositifs, outils et objets de gestion, ces derniers constituant les éléments de l'apprentissage.

Ainsi, de nombreux travaux sur l'apprentissage organisationnel donnent à voir un processus fortement contraint d'un point de vue cognitif. Définissant l'apprentissage comme un processus de détection et de correction des erreurs, celle-ci étant entendue comme l'écart entre une intention et ses conséquences effectives, (Argyris 1995) envisage deux modalités principales de correction des erreurs. La première, qualifiée d'apprentissage "en simple boucle" consiste à modifier le comportement des individus, leurs stratégies d'action, sans questionner les valeurs ou les croyances qui les sous-tendent. Le processus d'appropriation s'avère dès lors incomplet : la règle, le dispositif, l'outil ou l'objet de gestion sont mobilisés par le dirigeant, mais sans que cela modifie ses représentations et sans qu'il en use pour expliciter ses raisonnements ou le bien fondé des attributions causales qu'il défend. L'apprentissage "en double boucle" consiste à modifier le "programme maître", c'est-à-dire à changer les valeurs et les croyances qui guident les stratégies d'action. Argyris met en évidence les mécanismes cognitifs de protection chez l'individu - les routines défensives - et la manière dont ils obèrent les processus d'apprentissage en "double boucle". Ces routines défensives se manifestent dans l'écart entre les théories professées - les valeurs et idéaux dont nous pensons qu'ils gouvernent nos actions, et les théories utilisées, celles que nous mobilisons effectivement dans le cours de l'action. Elles poussent également l'individu à considérer que les causes de ses problèmes ne lui sont pas directement imputables, le conduisant ainsi à des attributions erronées. Les théories en usage produisant au niveau individuel des stratégies d'esquive et de dissimulation, elles peuvent, à l'échelle de l'organisation, déboucher sur une myopie stratégique, empêchant tout processus d'acquisition de nouvelles connaissances.

Argyris explique par la difficulté à réunir les conditions d'un apprentissage à double boucle la préférence des dirigeants pour le maintien du statut quo et la stabilité organisationnelle. La cartographie cognitive individuelle, dans cette optique, constitue un objet de gestion précieux pour aider les dirigeants à expliciter leurs représentations, faire sens de leurs actions, en dévoiler les ressorts profonds. Si les méthodologies de construction des cartes cognitives sont variées ${ }^{126}$, toutes postu-

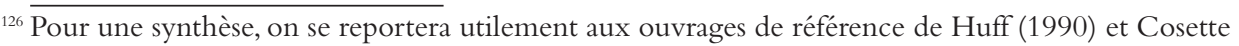
(1994). 
lent néanmoins la possibilité d'accéder aux représentations des individus et de les extraire sous une forme analysable. Elément de compréhension voire de dévoilement des routines ou croyances fondamentales de l'organisation cartographie cognitive collective), les cartes cognitives constituent ainsi un levier d'appropriation de la stratégie. Laroche (2001) suggère toutefois d'insérer la démarche cartographique dans une problématique précise et élaborée.

A un niveau individuel, la perspective rationnelle s'intéresse aux processus mentaux à l'œuvre dans une décision individuelle ou à la personnalité des décideurs. Elle montre le poids des représentations sociales dans les processus de formation des stratégies. Ainsi le dirigeant n'aborde-t-il jamais les situations stratégiques d'un œil vierge, mais a-t-il des "représentations de ce qui est important ou non, et de ce qu'il convient de faire ou non" (Laroche, 1993). Au niveau groupal, les travaux en psychosociologie montrent comment les interactions entre acteurs vont conditionner le processus d'appropriation, soit dans le sens d'une radicalisation, soit dans le sens du conformisme.

Ainsi, l'approche psychologique et psychanalytique des organisations, portée notamment par Manfred Kets de Vries, entend démontrer la dimension psychique de la vie organisationnelle. Pour Kets de Vries et Miller (1985), l'action des individus n'est pas guidée uniquement par la poursuite de buts rationnels, mais englobe également des composantes affectives, émotionnelles, inconscientes. L'auteur met ainsi en exergue l'incidence des processus mentaux et schèmes cognitifs des cadres dirigeants sur différents aspects organisationnels : prise de décision, structure, culture, processus collectifs... Se réclamant d'une démarche compréhensive fondée largement sur des études de cas, Kets de Vries va notamment s'intéresser aux pathologies et névroses du dirigeant, et à leurs conséquences organisationnelles. D'autres travaux, d'inspiration psychologique, soulignent le rôle de l'intuition dans la cognition managériale (strategic insight). Dans le champ de la stratégie, soulignent Atamer et Calori (1998), l'intuition a d'abord été vue comme un processus de reconnaissance et de capitalisation sur les expériences passées (Simon, 1965). Le concept d'intuition connaît par la suite une phase de vulgarisation, avec notamment les travaux de Tichy et Devanna (1986) sur le leader visionnaire, par opposition au leader instrumental. La vraie réhabilitation du concept peut néanmoins être datée de 1994, avec simultanément la critique de la planification stratégique opérée par Mintzberg (1994), et la controverse initiée par Hamel et Prahalad (1990) à l'encontre des modèles orthodoxes du management stratégique. Miller (1996) renvoie à un mode d'apprentissage émergent, holiste, par lequel les managers reconnaissent dans le flux des événements et des décisions, des "configurations" permettant d'articuler dans un système cohérent compétences actuelles et potentialités futures, choix stratégiques et organisation. L'intuition peut ainsi aider à l'identification des ressources et compétences clés, au repérage des sources d'avantage concurrentiel ou bien encore à l'identification des synergies entre départements. 


\section{Management stratégique et dynamiques d'appropriation des outils de gestion : proposition d'une grille de lecture.}

Alors que le champ de l'apprentissage organisationnel marque parfois une polarisation excessive sur le rôle du dirigeant, la notion de communauté de pratique permet d'envisager la stratégie comme une création et une mise en forme collectives. Une communauté de pratique est définie comme un réseau d'individus socialement liés, engagés dans une activité, une pratique conjointe, partageant un même langage, des mêmes préoccupations, une même passion, des mêmes concepts et qui développent leurs compétences par l'échange et des activités communes de résolution de problèmes. Ainsi, ne peut-on pas considérer que les managers responsables de filiales, de divisions, de directions métiers... constituent une communauté de pratique à géométrie variable, cherchant à concrétiser localement les visions du noyau stratégique ? Cette perspective s'inscrit dans l'évolution récente des démarches de planification stratégique (Martinet, 2001), centrées sur une logique d'animation, visant à faire émerger les actions stratégiques. La responsabilité de la planification stratégique passe de fait des fonctionnels aux opérationnels, du niveau central au niveau local. Ateliers de décision stratégiques, groupes de projet, groupes d'amélioration de la qualité fondent ici un dispositif de gestion au service de la communauté de la pratique, privilégiant un usage heuristique d'outils de gestion divers : matrices, scénarios, benchmarking, repérage des compétences fondamentales... L'appropriation de la stratégie, tant dans sa formation que sa mise en œuvre, prend alors le détour de l'expérimentation dans le cadre d'un processus davantage émergent et incrémental (Quinn, 1980). Projets et initiatives locales sont ainsi conduits en autonomie par les unités opérationnelles sur la base d'une intention stratégique exprimée par le noyau, qui soit suffisamment floue et ambiguë pour mettre en mouvement l'organisation. Ce processus de changement construit n'exclut pas le retour à des formes plus délibérées visant à stabiliser l'intention stratégique et le cadre de la nouvelle organisation. Cette logique d'appropriation entend se démarquer d'une polarisation excessive sur le rôle du dirigeant. Elle n'échappe pas néanmoins au risque de fragmentation des apprentissages (Kim, 1993), l'articulation des initiatives locales avec la stratégie globale étant problématique.

S'agissant de l'interaction entre le noyau stratégique et la périphérie, Simon (1965) a pu montrer comment les dirigeants orientaient l'attention des autres acteurs organisationnels sur certains aspects des situations stratégiques, par la configuration singulière des canaux de communication et les mécanismes d'allocation des ressources. Simons (1994) prolonge cette perspective en montrant comment les dispositifs de contrôle - levers of control - contribuent à cette focalisation de l'attention. Occasio (1997) va jusqu'à développer une vision de la firme fondée sur la mise en œuvre de mécanismes de sélection de l'attention "Attention-BasedView of the Firm"). Le rôle des routines organisationnelles comme moteur de l'attention a également été bien mis en évidence par Nelson et Winter (1982). Répertoire culturel des actions alternatives possibles permettant aux managers d'appréhender les principales issues, problèmes et opportunités stratégiques, les routines sont ainsi une réponse au problème de la rationalité limitée. Ce répertoire de routines est façonné sans être totalement déterminé par les ressources de l'entreprise. 
Si l'on entend la stratégie comme un processus permanent de construction et de reconstruction du sens par les acteurs (Weick, 1995), il apparaît dès lors aussi important de produire une stratégie "techniquement" efficace qu'une stratégie acceptable, légitime pour les différentes parties en présence - les stakeholders (Chanal, Lacroux et Mounoud, 2001). Les conversations stratégiques contribuent ainsi à l'émergence, au déploiement mais aussi à l'appropriation ou la clôture de la stratégie. Ces conversations, envisagées comme des interactions spontanées, s'appuient sur un répertoire partagé dans lequel puisent les différents membres, et agrégeant des objets de gestion divers : matrices et outils de diagnostic stratégique, plans stratégiques antérieurs, éléments de veille concurrentielle, cartes cognitives, mais aussi symboles lieux où se déroulent les conversations stratégiques, rituels, éléments de langage.

\subsection{L'appropriation comme processus collectif dynamique}

Développer une perspective politique sur l'appropriation de la stratégie par le noyau stratégique amène à s'intéresser au gouvernement des entreprises. Charreaux (1997) le décrit comme "l'ensemble des mécanismes organisationnels qui ont pour effet de délimiter les pouvoirs et d'influencer les décisions des dirigeants, autrement dit, qui "igouvernent " leur conduite et définissent leur espace discrétionnaire". La contribution du conseil d'administration à la stratégie de l'entreprise constitue à cet égard un terrain d'étude privilégié (Le Joly, 1998). Les divergences d'intérêts entre dirigeants et actionnaires nourrissent, en effet, le débat sur les orientations stratégiques de la firme. Choix d'alliances, choix technologiques, choix de diversification... constituent des options porteuses de tels conflits. Plusieurs travaux empiriques (Godard, 1996) soulignent l'incidence de la stratégie suivie sur la composition du conseil d'administration. La cooptation d'administrateurs externes peut ainsi être envisagée comme un moyen de s'attacher les contacts qui faciliteront l'accès aux capitaux nécessaires au développement de son activité (Le Joly, Ibid.), ou l'aideront à construire sa légitimité au regard de son environnement. Pour autant, on ne saurait sous-estimer le risque de perte d'autonomie stratégique auquel expose le principe de la cooptation, la mission, les buts mêmes de l'organisation pouvant dès lors se trouver singulièrement affectés par le jeu des différents groupes de pression externes.

S'agissant de la périphérie, le courant de l'analyse stratégique porté par Michel Crozier et Erhard Friedberg (Crozier, 1963 ; Crozier et Friedberg, 1977 ; Friedberg, 1993) met en exergue le rôle central de l'incertitude dans les organisations, et donne à voir des acteurs "stratèges" dont les interactions impliquent un jeu continuel autour des règles. Parce qu'il est créateur de règles, le pouvoir contribue à la stabilisation des interactions, et rend possible l'action collective. JeanDaniel Reynaud (1988), avec la théorie de la régulation conjointe, fait également de l'activité de production de normes et de règles, qu'il dénomme "l'activité de régulation", un principe fondateur de l'action sociale, s'inscrivant ainsi dans une 


\section{Management stratégique et dynamiques d'appropriation des outils de gestion : proposition d'une grille de lecture.}

longue tradition sociologique. Il montre que les règles qui fondent l'interaction sociale sont produites par les acteurs eux-mêmes, et que la modification des règles fait partie intégrante des stratégies des acteurs, ces derniers tentant de changer ou de maintenir les règles du jeu à leur profit. Ainsi, selon Reynaud, c'est moins le respect des règles que leur modification qui constitue le ressort principal de l'action collective. Le propos de l'auteur s'inscrit dès lors délibérément dans une perspective "appropriative" : en effet, la règle n'est jamais donnée telle quelle, et quand bien même elle préexisterait aux acteurs, ces derniers ne la reçoivent jamais passivement, mais se doivent de la mobiliser en situation, en d'autres termes de se l'approprier, de la rendre propre à un usage. L'auteur, dans un entretien récent ${ }^{127}$, fournit à cet égard un exemple éclairant :

“Il est évident que nous n'inventons pas la plupart des règles que nous suivons. Mais nous ne les recevons pas passivement : il faut mobiliser la règle. Par exemple, mon voisin fume dans un compartiment non fumeur et cela me gêne. Il faut alors que je mobilise la règle : que je l'invoque, que je proteste, le cas échéant que je prenne les autres personnes à témoin ou que j'appelle le contrôleur. Autrement dit, je dois brandir la règle sans quoi il ne se passera rien. Il y a donc bien une appropriation nécessaire".

Reynaud esquisse à cette occasion quelques conditions d'appropriation des règles de gestion, dont on observera qu'elles peuvent être parfaitement transposées au niveau des dispositifs, outils et objets de gestion :

- La règle, pour être appropriée, doit être rapportée aux fins d'une action commune, d'un projet commun qui lui donne sens et rend possible l'action collective. En d'autres termes : la règle "est acceptée par les autres comme pouvant diriger cette action collective", et "permet d'atteindre l'objet de cette action collective".

- La règle comprend une part héritée, c'est-à-dire qu'elle s'incarne dans des dispositifs, des outils, des objets de gestion des règles juridiques, des procédés, des techniques.... Cette inscription permet une économie cognitive, c'est-à-dire une mobilisation de la règle, sans que les acteurs en aient nécessairement conscience.

- Il existe, dans toute organisation, deux types de régulations concurrents et complémentaires : les régulations de contrôle, dont l'élaboration est extrêmement explicite, ont vocation à prescrire les comportements requis ou souhaitables de même qu'à signaler les comportements proscrits. Or, la règle prescrite, "officielle", nécessairement "faillible" et lacunaire, ne saurait prétendre résumer les situations de gestion dans leur totalité. Cette incomplétude ouvre par

127 "Il n'y a pas de règles sans projet", Entretien avec Jean Daniel Reynaud, in P. Cabin (Coord.), Les organisations, Editions Sciences Humaines, 1999, p 259-266. 
conséquent la voie à des régulations autonomes développées par les acteurs eux-mêmes, qui s'inscrivent dans les failles de la régulation de contrôle et permettent à celle-ci de correctement fonctionner. C'est donc la régulation autonome qui permet à la régulation de contrôle d'être appropriée.

L'école française des conventions, née à la fin des années 80, regroupant sociologues et économistes, entend comprendre sur quelles bases se construit une logique collective dès lors que les actions individuelles s'inscrivent dans une pluralité de registres de justification. La question de l'appropriation, au niveau collectif, devient celle de la légitimité, ou plus précisément, des conflits de légitimité qui traversent la vie des organisations. Boltanski et Thévenot (1987) identifient ainsi six grands principes de légitimité concurrents, indifféremment dénommés "natures", "mondes" ou "cités" : la cité inspirée se réfêre à un principe d'innovation, de créativité ; la cité domestique est un univers de traditions, d'attachement et de respect des règles qui fondent la vie du groupe ; la cité de renom vise la reconnaissance sociale ; la cité civique fonde ses actions sur la recherche de l'intérêt général ; la cité marchande s'appuie sur des relations contractuelles et une logique donnant-donnant ; la cité industrielle, enfin, entend valoriser l'efficacité et la productivité. L'appropriation, pour opérer à un niveau collectif, suppose des accords, des compromis tacites ou explicites, entre ces différents principes de légitimité. L'appropriation d'une intention stratégique pourra emprunter à différents registres de justification : la légitimité civique i.e. l'accent sur le développement durable), la légitimité domestique la continuité avec la culture, les routines de l'organisation), la légitimité inspirée i.e. la recherche de rupture, la volonté affichée de transformer les règles du jeu concurrentiel), la légitimité industrielle i.e. la recherche d'une articulation optimale entre stratégie globale et stratégies fonctionnelles).

Johnson et Scholes (2002), au travers de leur analyse du tissu culturel, font de ce dernier un des leviers principaux de tout projet de changement comme l'une des sources d'inertie majeure. Ils montrent que la régulation par la culture comme projet d'appropriation de la stratégie par le corps social suppose un travail simultané sur les composantes symboliques de la culture mythes, rituels et routines, symboles...), mais aussi ses composantes gestionnaires incidence de la culture sur les systèmes de contrôle, l'organisation interne, l'équilibre des pouvoirs). Schein (1985), pour sa part, montre à quel point la culture organisationnelle est indissociable de l'exercice de la fonction de leadership. Le leader aurait ainsi pour rôle de créer, de gérer, mais aussi le cas échéant de détruire la culture de l'organisation en fonction de ses propres postulats de base et de l'intention stratégique dont il estime être porteur. Le leader utilise à cet effet des mécanismes conscients et inconscients pour renforcer l'adhésion du corps social à la culture : focalisation de l'attention sur certains événements, critères de sélection et de promotion des salariés, structure organisationnelle, routines et procédures, histoires et légendes... 


\subsection{L’appropriation comme processus normalisé instantané}

La planification, au sens large, du travail et son exécution, ne peut être concentrée sur les mêmes individus. Convaincu par ce principe, Taylor (1913) scinde donc les planificateurs et les exécutants. Ainsi naissent les bureaux des méthodes où se concentrent les ingénieurs, le cœur du noyau stratégique des entreprises industrielles de l'époque. Leur fonction est de développer des méthodes scientifiques pour l'accomplissement des tâches, fixer les objectifs de productivité, établir des systèmes de primes de rendement, éduquer le personnel pour l'utilisation de ces méthodes de travail et la réalisation des objectifs. Taylor en arrive à mettre en évidence quatre grands principes qui caractérisent son système de management :

- Il appartient aux membres de la Direction comprenez du management, de l'encadrement), de mettre au point la technique d'exécution de chaque élément du travail, les ouvriers ayant seulement à perfectionner les outils et les conditions de travail. Les membres de la Direction doivent donc réunir toutes les connaissances empiriques, les classer et les transformer en lois scientifiques. C'est à eux que revient la définition des conditions optimales d'exécution d'une tâche et la fixation des normes du travail aux pièces.

- Les ouvriers doivent être sélectionnés puis entraînés, afin de perfectionner progressivement leurs qualités et leurs connaissances.

- Les ouvriers doivent suivre les directives données sur la façon d'exécuter le travail. Taylor a la certitude que le travail doit être exécuté conformément aux règles découvertes par la Direction qui a dû mettre au point les meilleures méthodes avec les outils les plus appropriés.

- Dans ces conditions, la responsabilité du travail est donc vraiment partagée entre les ouvriers et les membres de la Direction.

Les apports de Taylor et Fayol sont fréquemment mis en parallèles : de fait, Henri Fayol apporte dans le domaine administratif une contribution tout à fait comparable à celle que Taylor propose pour l'activité de production. Plus pragmatique que déterministe, Fayol (1916) énonce cinq principes aujourd'hui célèbres, significatifs de la perspective rationnelle d'appropriation des objets et outils de gestion :

- Le commandement assure la bonne marche de l'organisation et la direction des hommes y tient une place essentielle. C'est une activité qui repose à la fois sur la personnalité du dirigeant et sur sa connaissance de l'administration de l'entreprise.

- Le contrôle consiste à vérifier l'application du programme d'action, des procédures et des ordres. Inséparable des sanctions, il fait l'objet lui-même de procédures rigoureuses. 
- La prévision doit permettre de préparer l'avenir en établissant un programme à la fois souple pour rester adaptable aux variations et suffisamment précis pour servir de base commune, et éviter toute confusion, aux différents acteurs. C'est une tâche qui fait appel à la créativité autant qu'au calcul.

- L'organisation consiste à munir l'entreprise des organes nécessaires à son fonctionnement, à définir leurs fonctions, leurs responsabilités, établir des procédures.

- La coordination vise à relier, unir et harmoniser les efforts de tous, principalement au travers de conférences hebdomadaires. Certains cadres de direction sont invités à y participer.

Les théories de la micro-économie occupent également une place importante dans les processus de formation des stratégies endogènes par les acteurs du noyau stratégique. Ainsi peut-on évoquer la contribution de Coase (1937), considéré comme l'un des pionniers des théories de la firme. Les économistes classiques se focalisaient en effet sur les marchés et leurs mécanismes. Ils laissaient par contre de côté les firmes, jugées comme des boîtes noires dans lesquelles entraient des ressources et ressortaient des biens. Certains les ont tout de même évoqués, mais sans réellement se pencher sur le fait qu'à l'intérieur d'une firme, c'est un entrepreneur qui assure la coordination et non un mécanisme de prix : Marshall considérait l'organisation comme un facteur de production ; Adam Smith attribuait à l'entrepreneur un rôle d'organisateur de la division du travail. Ronald Coase fut le premier à s'intéresser de façon importante à la firme. Il avança le fait que les transactions avaient un coût qui justifiait la création d'une firme. Outre l'attrait "d'être son propre maitre", l'entrepreneur décide donc d'avoir recours à la firme pour des raisons économiques. Williamson (1975) a repris et adapté cette économie des transactions, afin d'une part de rendre opérationnel le concept de coût de transaction, et d'autre part d'analyser la répartition des transactions entre le marché et la hiérarchie selon les éléments comportementaux. Dès lors, les apports de Williamson, ainsi que les travaux qui lui ont succédé, nous éloignent d'une perspective purement rationnelle, envisagée d'un point de vue stricto sensu.

Eu égard au rôle prédominant de la régulation de contrôle et donc du noyau stratégique) dans une approche rationnelle d'appropriation de la stratégie en entreprise, on pourrait s'attendre à ce que les acteurs de la périphérie soient absents des débats. S'il est vrai qu'aucun courant théorique n'abonde en ce sens, on peut toutefois évoquer le rôle que joue le plus souvent la périphérie comme relais de la régulation de contrôle. Ainsi, si l'on s'en tient à une approche déterministe en termes de mode de gouvernement principal des organisations le commandement), Martinet (2001) revient sur les principaux traits de la planification : imposer les stratégies centrales et contrôler les unités, le tout dans une logique hiérarchique descendante reposant sur une gestion par les règles. Les acteurs de la périphérie 
interviennent alors comme un appui opérationnel à la mise en œuvre des grandes lignes d'une planification stratégique centralisée autour des décideurs du noyau stratégique.

De même, les approches classiques de la recherche opérationnelle peuvent également être évoquées dans cette perspective, la périphérie utilisant la modélisation mathématique pour formuler et analyser rigoureusement les problèmes de décision complexes auxquels peuvent être confrontés les membres du noyau stratégique (Roy, 1997).

S'agissant enfin de l'interaction entre noyau stratégique et périphérie, il convient de souligner que la tension entre exploitation et exploration (Levinthal et March, 1993), organisation et innovation, compétences actuelles et compétences latentes ou potentielles, constitue assurément un moteur des processus de formation des stratégies endogènes. Comme le notent justement (Métais et Tannery 2001) :"en créant un écart sur les ressources actuelles et présentes, notamment par des effets de mise en scène et d'image sur les futurs désirés, la tension contribue à l'institution d'une architecture stratégique". Le concept d'intention stratégique Hamel et Prahalad, Ibid.) est au centre de la gestion de cette tension, qui exprime les compétences dont l'entreprise entend se doter à l'avenir. L'appropriation de la stratégie par les autres acteurs organisationnels sera d'autant facilitée que ceux-ci pourront, sur la durée, faire le lien entre la situation présente et un futur désiré (Métais et Tannery, Ibid.).

\section{Conclusion : vers un nouveau couple ago-antagoniste ?}

Si le recours au couple management stratégique exogène / management stratégique endogène est une manière commode d'éclairer les processus de décision stratégiques et la manière dont ils sont appropriés par les acteurs, le clivage pourrait apparaître à bien des égards artificiel, voire caduc. Ainsi l'opposition management stratégique exogène / management stratégique endogène ne serait qu'un avatar des multiples couples ago-antagonistes qui traversent le champ du management stratégique : interne / externe, pensée / action, formulation / mise en œuvre, contenu / processus ou rationalité substantive / rationalité procédurale dans les termes de Simon), délibéré / émergent, intégration / différenciation ... Les modèles rationnels qui fondent l'orthodoxie du management stratégique se sont eux-mêmes développés sur la base de dichotomies. Le modèle de Harvard, l'analyse SWOT, les matrices de gestion de portefeuille s'appuient ainsi largement sur la dichotomie interne/externe. Ainsi, la tentation est grande d'analyser le couple management stratégique exogène / management stratégique endogène à l'aune de ces catégories. La logique exogène renverrait ainsi à un déterminisme s'exerçant sur les choix stratégiques en affirmant le primat de l'environnement externe ; la logique endogène réhabiliterait a contrario le rôle de l'intentionnalité 
en stratégie et le primat de l'environnement interne. Il y a là un faux-semblant qui doit être dénoncé, tant le couple management stratégique exogène / management stratégique endogène transcende les catégories précédemment évoquées.

Ainsi, sur le versant exogène et sur le couple déterminisme / intentionnalisme :

La théorie institutionnelle définit un acteur stratégique qui n'est pas un acteur strictement rationnel, mais qui est aussi inscrit socialement dans un contexte, un environnement institutionnel lui prescrivant des façons légitimes d'agir. La sociologie institutionnelle explique la survie de l'entreprise par la conformation à ces normes, seules à même de lui conférer la légitimité nécessaire pour accéder aux ressources qui lui font défaut : salariés, réputation (Di Maggio et Powell, 1983). Pour James March, nos capacités de cadrage et d'interprétation des situations stratégiques sont limitées par un biais ethnocentrique, qui nous empêche de voir au-delà des conventions de notre milieu. De la même manière, l'écologie des populations postule que l'environnement est le moteur d'un processus à long terme de sélection des formes organisationnelles, les entreprises n'ayant d'autre choix de se conformer, s'adapter à ce processus ou disparaitre. Pour autant, doit on en conclure à une stricte détermination des choix stratégiques? Pas nécessairement si l'on renverse la proposition, et si l'on considère que cet acteur peut aussi se comporter comme un entrepreneur institutionnel, dont la stratégie vise à changer les règles du jeu établies (Laroche et Nioche, 1998). On rappellera dès lors avec (Desreumaux 1996) que "les dirigeants ont toujours, au moins au plan des principes, le choix d'une action sur l'organisation ou d'une action de modification du contexte".

De fait, la tentation visant à associer moteurs endogènes de la stratégie avec l'idée de volontarisme, comme celle rapportant l'idée de déterminisme aux moteurs exogènes du pilotage stratégique, doit être dénoncée comme sommaire et non fondée. Desreumaux (1996) souligne très justement que "le déterminisme peut être autant interne qu'externe à travers le jeu des routines, des traditions, et de l'ensemble des facteurs structurels, culturels, psychologiques et autres qui pèsent de façon non " consciente " sur le choix des décideurs". La notion de path dependency, prégnante dans la théorie des ressources, ne donne-t-elle pas à voir un processus d'évolution de l'entreprise borné par les choix stratégiques et investissements initiaux, de même qu'elle souligne l'inertie structurelle et le poids des routines face aux mécanismes de réallocation des ressources?

De même, sur le versant endogène et sur le couple émergent / délibéré :

Contrairement à ce que pourrait laisser entendre une association trompeuse, le versant endogène du pilotage stratégique n'implique pas nécessairement un processus de formation des stratégies émergent. Le caractère émergent ou délibéré du processus dépend ainsi moins d'un clivage exogène/endogène, que du degré de formalisation de l'intention stratégique initiale (Grimand et Vandangeon- 
Derumez, 1999), des modes de gouvernance dominants (Martinet, 2001), des difficultés d'articulation global/local, ou bien encore de l'ampleur de la réorientation stratégique visée.

Dès lors, en quoi le couple exogène / endogène constitue-t-il un couple novateur permettant d'éclairer de manière pertinente l'étude des dynamiques d'appropriation des outils de gestion dans le champ de la stratégie ? Nous avançons les éléments de réponse suivants :

- il donne à voir des leviers et des trajectoires différents d'appropriation de la stratégie, tant pour ses concepteurs que pour les utilisateurs finaux, selon que l'on se situe sur le pôle exogène ou endogène du pilotage stratégique ;

- il intègre dans la démarche stratégique une réflexion sur la nature des situations stratégiques en cause moments "chauds" versus périodes de "normalité" stratégique ;

- il permet d'intégrer l'ensemble des parties prenantes, internes comme externes, dans la réflexion stratégique ;

- il englobe les différentes rationalités à l'œuvre psycho-cognitive, socio-politique, rationnelle dans tout processus de changement stratégique et d'apprentissage ;

- il accorde une place singulière aux objets de gestion, qu'ils soient institutionnels ou organisationnels, dans les processus de formation et d'appropriation des stratégies.

Au final, plutôt que de poser le débat en termes d'alternative l'action stratégique ne peut à notre sens s'inscrire exclusivement dans un registre endogène ou exogène, nous invitons à envisager leur complémentarité et une gestion des équilibrations entre ces deux pôles. C'est dans cette perspective que nous appelons au développement de travaux empiriques qui restent à ce jour largement à venir, comme par exemple concernant l'étude des conditions de l'appropriation de l'intention stratégique envisagée comme dispositif de pilotage Grimand et (Goy, 2005), et plus généralement sur la place des dynamiques endogènes et exogènes dans les changements stratégiques.

\section{Bibliographie}

N. Alter (1990), La gestion du désordre en entreprise, L'Harmattan, Paris.

H.I. Ansoff (1979), Strategic Management, Macmillan, London.

M.S. Archer (1995), Realistic Social Theory: the Morphogenetic Approach, Cambridge university press, $354 \mathrm{p}$. 
C. Argyris (Ed.) (1995), Savoir pour agir : surmonter les obstacles à l'apprentissage organisationnel, Interéditions, Paris.

T. Atamer, R. Calori (1998), Diagnostic et décisions stratégiques, Dunod Paris.

N. Aubert, V. De Gaulejac (1991), Le Coût de l'Excellence, Seuil.

L. Boltanski, L.Thévenot (1987), Les économies de la grandeur, PUF, Paris.

P. Bourdieu (2000), Esquisse d'une théorie de la pratique, Essais, première édition en 1972.

V. Chanal, F. Lacroux, E. Mounoud (2001), "Vers une approche ingénierique des conversations stratégiques", In : Faire de la recherche en management stratégique, Vuibert, Paris.

G. Charreaux (1997), Le gouvernement des entreprises. Corporate Governance. Théorie et Faits, Economica, Paris.

J. Child (1997), "Strategic choice in the analysis of action, structure, organizations and environment: retrospect and prospect", Orgnizations Studies, vol. 18, n¹, p. $43-76$.

R.H. Coase (1937), The nature of the firm, Economica.

P. Cosette (Dir) (1994), Cartes cognitives et organisations, Presses de l'Université de Laval/Eska, Laval.

D. Courpasson (2000), L'action contrainte. Organisations libérales et domination, PUF, Paris.

M. Crozier (1963), Le phénomène bureaucratique, Paris.

M. Crozier, E. Friedberg (1977), L'acteur et le système. Les contraintes de l'action collective, Seuil, Paris.

F.X. De Vaujany , Coord., (2005), De la conception à l'usage : éléments d'un management de l'appropriation des objets et outils de gestion, Editions EMS, Collection "Questions de Société".

G. Dechamp, H. Goy, A. Grimand, T. Lévy, F.X. de Vaujany (2005), “Gestion stratégique et perspective appropriative : comparaison des modes endogènes et exogènes", dans F-X. De Vaujany (coord.), De la conception à l'usage : éléments d'un management de l'appropriation des objets et outils de gestion, Editions EMS, Collection "Questions de Société “.

A. Desreumaux (1996), "Nouvelles formes d'organisation et évolution de l'entreprise", Revue Française de Gestion, n¹07, p.86-108.

P. Di Maggio, W. Powell (1983), The Iron-Cage Revisited : Institutional Isomorphism and Collective Rationality in Organizational Field, American Sociological Review, Vol. 48, p.147-160.

E. Enriquez (1992), L'organisation en analyse, PUF.

H. Fayol (1916), “Administration industrielle et générale”, Bulletin de la Société de l'Industrie Minérale, n¹0, p.5-164.

E. Friedberg (1993), Le Pouvoir et la Règle, Seuil.

C.J.G. Gersick (1994), "Pacing strategic change : the case of a new venture", Academy of Management Journal, Vol 37, n¹, pp. 9-45. 
A. Giddens (1984), The Constitution of Society: Outline of a Theory of Structuration, University of California press.

L. Godard (1996), Conseil d'administration, stratégie et performance, Thèse pour le doctorat en Sciences de Gestion, Université de Bourgogne.

A. Grimand, H. Goy (2005), "De la genèse de l'intention stratégique à son appropriation", Présentation en session interactive, $14^{\text {ème }}$ Conférence de l'Association Internationale de Management Stratégique AIMS), Angers, 0709 juin, 23p.

A. Grimand, Vandangeon-Derumez I. (1999), "L'organisation qualifiante entre autonomie et changement : vers une nouvelle conduite des processus de changement”, Revue de Gestion des Ressources Humaines, n³2, p.108-126.

G. Hamel, C.K. Prahalad (1990), "The Core Competence of the Corporation", Harvard Business Review, n³, p.79-91.

A.S. Huff (Ed) (1990), Mapping Strategic Thought, Chichester, Wiley.

G. Johnson, H. Scholes H. (2002), Stratégique, Pearson Education, $2^{\text {ème }}$ édition.

M. Kets de Vries, D. Miller (1985), L'entreprise névrosée, Mac Graw Hill Traduction française), Paris.

D.H. Kim (1993), "The Link Between Individual and Organizational Learning”, Sloan Management Review, vol.35, n¹, p.37-50.

H. Laroche (1993), "Les décisions stratégiques ou la petite fabrique de la stratégie”, Sciences Humaines, hors-série n², repris dans P. Cabin Coord.) 1999), Les organisations, Editions Sciences Humaines, p.133-138.

H. Laroche (2001), "Les approches cognitives de la stratégie" in Martinet A.-Ch. et R. A. Thiétart, Stratégies. Actualité et futurs de la recherche, Vuibert, Fnege, p.101-114.

H. Laroche, J.P. Nioche (1998), "Les nouveaux fondements de la stratégie”, in Laroche et Nioche Dir.), Repenser la stratégie. Fondements et perspectives, Vuibert, Institut Vital Roux.

K. Le Joly (1998), “Le conseil d'administration : contrôleur ou stratège ?”, In : Martinet A. Ch. et R. A. Thiétart, Stratégies. Actualité et futurs de la recherche, Vuibert, Fnege, p.111-132.

D.A. Levinthal, J. March (1993), “The Myopa of Learning”, Strategic Management Journal, Vol. 14, p.95-112.

A.C. Martinet (1984), Management stratégique : organisation et politique, McGrawHill.

A.C. Martinet (2001), "Le faux déclin de la planification stratégique" In : Martinet A. Ch. et R. A. Thiétart : Stratégies. Actualité et futurs de la recherche, Vuibert, Fnege, p.175-193.

E. Métai, F. Tannery (2001), “La création de la stratégie : éléments de repérage et propositions théoriques" In : Martinet A. Ch. et R. A. Thiétart : Stratégies. Actualité et futurs de la recherche, Vuibert, Fnege, p.115-138. 
D. Miller (1996), “A Preliminary Typology of Organizational Learning : Synthesizing the Literature", Journal of Management,Vol. 22, n³, p.485-505.

H. Mintzberg (1994), Grandeur et décadence de la planification stratégique, Dunod, Paris.

G. Morgan (1997), Images of Organization, Sage, Thousand Oaks.

R. Nelson, S.G. Winter (1982), An Evolutionnary Theory of Economic Change, Cambridge, Harvard University Press.

W. Occasio (1997), "Towards an attention-based view of the firm", Strategic Management Journal, vol.18 special issue), p.187-206.

M. Pagès, M. Bonetti, V. de Gaulejac, D. Descendre (1992), L'emprise de l'organisation, PUF, première édition en 1992.

J. Piaget (1967), Biologie et connaissance : essai sur les relations entre les régulations organiques et les processus cognitifs, Gallimard, Paris.

J. Piaget (1975), L'équilibration des structures cognitives : problème central du développement, Presses Universitaires de France, Paris.

M.E. Porter (1985), Competitive Advantage, New York : Free Press.

J.B. Quinn (1980), Strategies for Change : Logical Incrementalism, Homewood, IL : Irwin.

J.D. Reynaud (1988), "Les régulations dans les organisations : régulation de contrôle et régulation autonome", Revue française de sociologie, XXIX, pp. 5-18.

B. Roy (1997), “La recherche opérationnelle entre acteurs et réalités”, Annales des Mines, Gérer et Comprendre, n47, p.16-27.

R. Sainsaulieu et al. (1998), Les mondes sociaux de l'entreprise, Eds. Desclée de Brouwer, Paris.

E.H. Schein (1985), Organizational Culture and Leadership, Jossey-Bass, San Francisco.

P. Senge (1990), “The leader's new work : building learning organization”, Sloan Management Review, Fall, p.7-22.

H.A. Simon (1965), Administrative Behavior, 2nd. Ed.), New York: Free Press.

R. Simons (1994), "How New Top Managers use Control Systems as Levers of Strategic Renewal”, Strategic Management Journal, vol. 15, p.169-189.

F.W.Taylor (1913), The Principles of Scientific Management, Harper \& Row, New York.

N.M. Tichy, M.A Devanna (1986), The Transformational Leader, Wiley, New York.

K.E. Weick (1995), Sensemaking in Organizations, Sage Publications.

O.E. Williamson (1975), Markets and Hierarchies: Analysis and Antitrust Implications, Free Press, New York. 